\title{
An electronic cine camera system for the automatic collection and analysis of high-speed movement of unrestrained animals
}

\author{
CHRIS M, WIELAND and ROBERT C. EATON \\ University of Colorado, Boulder, Colorado
}

\begin{abstract}
A system is decribed that collects visual data using a solid-state photodiode image sensor at rates of up to 600 frames/8ec in a binary format suitable for image-analysis techniques. Of particular interest is the system's ability to analyze high-speed movement of animals without prior modification of the subject (e.g., attachment of lamps or reflectors to the subject).
\end{abstract}

Even before the development of the cine camera, early investigators engaged in the analysis of animal movement by photographic means. Using a bank of up to 24 still cameras, with shutters triggered to open in a sequential fashion, Muybridge (1887/1979) began a new field of biological study by collecting and publishing numerous photographs that detailed for the first time the locomotion of man and animals.

Since then, quantitative analysis of movement has been improved substantially by the development of the cine camera. However, extraction of useful data from cine films has remained a time-consuming task, requiring tedious frame-by-frame measurements usually done by hand. The development of microcomputers and various associated peripherals (digitizers, $\mathrm{X}-\mathrm{Y}$ plotters) has eased the strain of hand analysis, but still requires the investigator to view each frame of the film, marking points of interest. This is particularly problematic in applications requiring high-speed film analysis (e.g., Eaton, Lavender, \& Wieland, 1981).

Recently developed techniques have included one in which a light source or reflector is attached to a subject under study and the $\mathrm{X}, \mathrm{Y}$ coordinates of these light sources are automatically recorded and made available for direct computer analysis and on-line storage (Brugger \& Milner, 1978; Rudell, 1979). Typically, such a system is used to record the joint positions of moving people or animals during performance of a particular motor pattern. This may allow a description of the functional development of limb use during ontogeny or after injury (Amassian \& Rudell, 1978) or may be used in making recommendations to increase the efficiency of an athlete's performance (Winter, Hobson, \& Greenlaw,

This project was supported in part by Grant RR07013-17, awarded by the Biomedical Research Support Grant Program, Division of Research Resources, NIH, and by grants from the National Science Foundation (Grants BNS79-005570 and BNS81-12423). The authors' mailing address is: Behavioral Biology Group, Department of Biology, E.P.O., University of Colorado, Boulder, Colorado 80309.
1972). For certain applications, it is difficult or inconvenient to mount light sources on the subject; a less obtrusive technique is required to record and analyze movement. In addition, in systems based on a videocamera (Rudell, 1979), imaging is limited to a frame rate of $60 \mathrm{~Hz}$, or about $17 \mathrm{msec}$ between frames. This does not allow for fine temporal resolution of rapid movements. Furthermore, because of the limited frequency response of the Vidicon's photosensitive element, a residual image remains for several frames after an object has moved to a new position. This characteristic, known as discharge lag, complicates the accurate tracking of quickly moving objects when video-based systems are used (Hall, 1971).

Here, a technique of motion analysis is described that in many applications involves no attachments to, or modifications of, the subject under study. This system allows an animal to move freely and permits motion analysis of animals whose habitat or small size precludes the attachment of a light source. Furthermore, the frame-taking rate is about 10 times higher than that of most available video systems, and the frequency response of the photodiode image sensor is fast enough to eliminate the problem of residual images. The cost of the system is comparable to, or less than, that of cine film cameras that operate at similarly high frame-taking rates. A preliminary account of this system is noted elsewhere (Wieland \& Eaton, 1983).

\section{TECHNICAL DESCRIPTION}

The system is based on a commercially available solid-state image-sensing unit (MC521/RS521) manufactured by EG\&G Reticon (345 Potrero Avenue, Sunnyvale, California 94086). This device uses a square matrix of $100 \times 100$ photodiodes as its image-sensing element and delivors a digital (binary) reading from each of the 10,000 photodiodes in the matrix. Alternatively, analog readings may be obtained from each photodiode. The image to be analyzed is focused on the surface of 
the photodiode matrix by a standard $\mathrm{C}$-mount camera lens, and the picture is formed as the matrix is scanned in a raster pattern, with a reading being taken from each photodiode. Data are transmitted by the camera, one bit at a time, in a serial stream at rates of up to $6 \mathrm{MHz}$ (600 frames/sec). In the binary mode, the camera returns a logic " 1 " if the light striking a given photodiode is above the user-specified threshold; otherwise, a " 0 " is returned.

The data delivered by the camera are converted from serial to 8-bit parallel format and transferred to the semiconductor memory that has been added to the camera controller unit (Figure 1). The camera generates

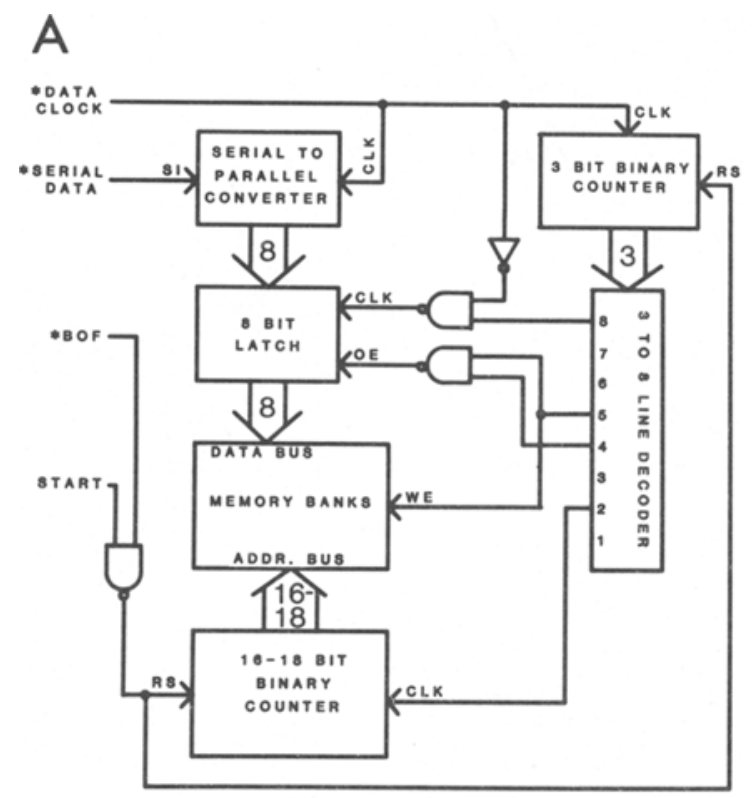

B

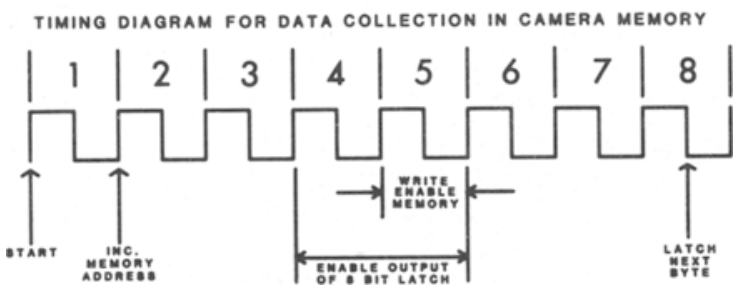

Figure 1. (A) Block diagram showing the circuitry that converts serial data from camera unit into a format suitable for transfer to a computer. Signals marked with * are generated by the Reticon camera unit. Abbreviations: ADDR-address, BOF-beginning of frame synchronization pulse, CLK -clock, OE-output enable, RS-reset, SI-serial data input, and WEwrite enable. The 16-18-bit binary counter supplying the memory bank address bus allows from one to four $64 \mathrm{~K}$ banks. (B) Timing diagram illustrating the sequence of data transfer from camera unit to semiconductor memory. Square wave is DATA CLOCK, and numbers above square wave represent sequence of 3 to 8 DECODER output. Actions indicated by vertical arrows occur at data clock transitions; actions shown with horizontal arrows occur for duration indicated by length of arrows. This eight-cycle sequence is repeated 1,250 times for transfer of each frame $(10,000$ bits $)$.

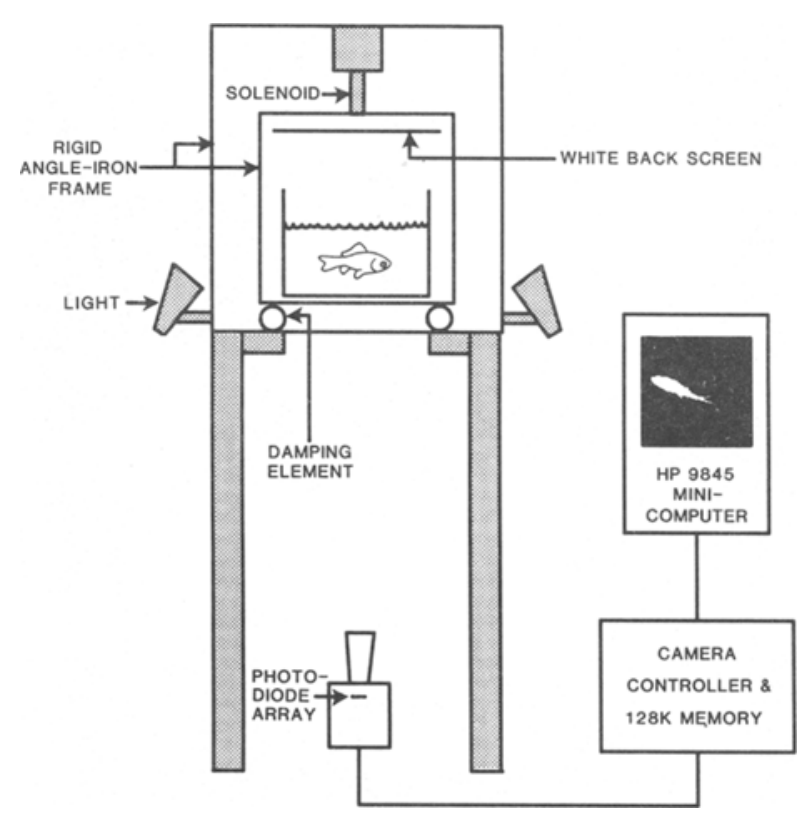

Figure 2. Diagram illustrating a complete system for recording and analyzing startle-response behavior in the goldfish. The solenoid is used to initiate the behavior by giving a small, but sudden, displacement of the aquarium (which is sitting on the base of the inner rigid frame). At the same time, the minicomputer triggers the camera controller to initiate data collection. Lighting of the back screen is accomplished by four $100-\mathrm{W}$ light bulbs mounted two on each side (only two are shown). The damping elements are 6.5 -cm-diam rubber balls.

1 bit for each photodiode and 10,000 bits for each frame. In 8-bit format, this requires $64 \mathrm{~KB}$ of memory for each 52 frames of data. Data are collected in the camera memory for the duration of the event under study, since transfer directly to a computer is difficult at the higher frame rates. The system is therefore designed to gather data in high-speed, short-duration bursts, and is not intended for real-time analysis. After data are gathered in the camera's memory, the information is transferred to a minicomputer (HewlettPackard 9845B) and is stored for analysis at a later time.

Image-analysis techniques are used in processing the data to obtain positional information. This avoids the need for mounting a light source on the animal. With our system, the animal is viewed by the camera against a lighted white background (Figure 2). As shown in Figure 3 , the photodiode matrix generates the animal's silhouette, in this case a fish in an aquarium, to which image-analysis techniques are applied. For some studies, one may merely require the position of the animal in two-dimensional space relative to a fixed location or to other animals. In this case, an algorithm that finds the centroid of each silhouette will return the proper information.

Other studies may utilize more conventional techniques and thus require the attachment of a light source to the object under study. For instance, in many studies, the $X, Y$ coordinates of individual limbs or joints may be 


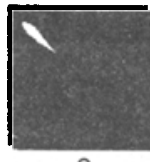

0

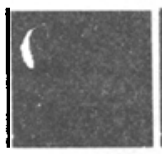

50

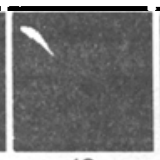

10

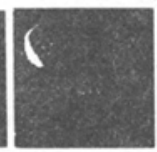

60

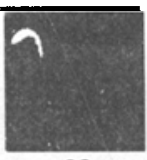

20

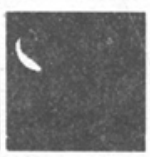

70

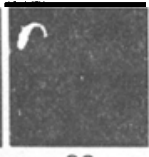

30

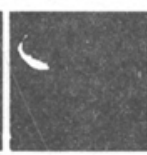

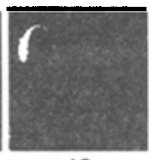

40

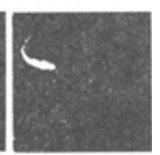

90
Figure 3. Selected frames (made with the electronic cine camera) of a $13 \cdot \mathrm{cm}$ goldfish performing a $\mathrm{C}$-ty pe startle response to an acoustic stimulus. Image-taking rate, 500 frames $/ \mathrm{sec}$. Numbers given below each frame indicate the time (in milliseconds) elapsed since application of the stimulus.

required, and, depending on the particular experimental animal being used, a silhouette may obscure limbs when they overlap the animal's body. Another problem can occur if certain areas of an animal's body reflect light much more efficiently than do others, such as in an animal with patches of light and dark fur. In such instances, the attachment of point light sources to individual limbs would be necessary to track their movements. In this application, the photodiode system has an advantage over the majority of video tracking systems, since the photodiode image detector is highly sensitive to infrared (IR) light. Thus, IR lamps or LEDs may be used in conjunction with lens filters for image recording in normal room lighting.

\section{CURRENT APPLICATION}

This system allows a highly sophisticated analysis of movement of subjects free from any external attachments such as lights or reflectors, for a number of applications. For example, image-analysis techniques for our particular application involve the accurate measurement of the motor performance of the fast-start behavior in the goldfish (Eaton et al., 1981). This startle response is easily demonstrated by tapping on the side of an aquarium

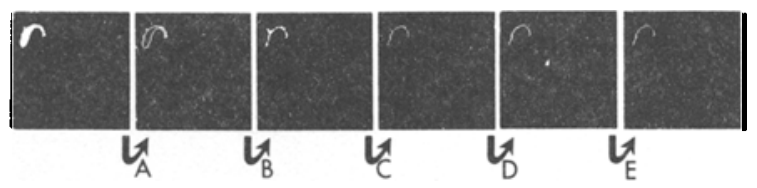

Figure 4. Sequence of steps in the analysis of the motion pattern. (A) Determination of the border of original figure and removal of "noise" pixels (those points not associated with figure under study). (B) Midline generation based on thinning algorithm. (C) Midline reduction to minimum possible number of points. (D) Removal of any unnecessary branches from midline. (E) Extension of endpoints of midline to limits of the original silhouette. Following the step in (F), the computer determines which end of the midline is the head and then saves the $X, Y$ coordinates of the pixels contained in the midline. The processing of a single image from (A) through (E) takes about 1 min with our Hewlett-Packard 9845 computer, using a combination of HP Extended BASIC and assembly language. containing a small fish. The animal will typically respond with a strong synchronous contraction of the musculature along one side of the body, bringing the animal into a "C" shape (Stage 1). Following this, the animal straightens its tail, propelling itself from its original position in the water (Stage 2). The two mechanical stages of this behavior have a duration of about 100 millisec and are illustrated by the images shown in Figure 3.

The results of the application of image-analysis techniques are shown in Figure 4 to illustrate the processing of the binary data from a single frame of Figure 3. Following this analysis, some of the measurements the computer is able to determine from the images are: (1) latency of initiation of movement; (2) displacement speed of the tip of the nose; (3) angular velocity of the turn; (4) direction of the turn (right or left); (5) distance traveled during the behavior; and (6) measurement of variability of the animal's performance ("stereotypy").

Such measurements may be used to detect any changes in the animal's motor performance that are due to experimental manipulations, or may be used in conjunction with neurophysiological data to uncover the mechanisms of neural control of certain aspects of the behavior.

\section{GENERAL APPLICATIONS}

This system was designed primarily for the recording of high-speed, short-term behaviors. As stated above, each $64 \mathrm{~K}$ of camera memory (cost $=$ about $\$ 300$ at the time of this writing) holds 52 images or frames, which, at $600 \mathrm{frames} / \mathrm{sec}$, is $86 \mathrm{msec}$ of activity, or a duration of $866 \mathrm{msec}$ at video frame rates $(60 / \mathrm{sec})$. To increase these times, memory can be easily added in $64 \mathrm{~K}$ increments according to the circuit design of Figure 1. Since image data must be collected in memory circuits before being transferred to a computer, it is not feasible to record long-term behaviors at high speeds. However, for many motor patterns, one to four $64 \mathrm{~K}$ banks of memory are sufficient for capturing the entire behavior. Schleidt (1974), for example, estimated the duration of most fixed-action patterns to be in the range 0.1 to $10.0 \mathrm{sec}$, with the average duration being around $1.0 \mathrm{sec}$.

One consequence of the high-frame-rate capabilities of this system is the lowered spatial resolution of the photodiode image sensor ( $100 \times 100$ pixels), which is about one-sixth the resolution typically available when using a video system (256 $\times 256$ pixels). In order to minimize this loss of resolution, the image of the subject being recorded should be focused onto as large a proportion of the imaging elements as possible. One method of accomplishing this is to mount the photodiode camera on a movable platform, so that it can be positioned directly over the subject just before recording, giving the maximum resolution. Another solution, which we employ in our study of fish startle responses, is to restrict the subject's activity to a limited area bounded by a 
transparent Plexiglas wall corresponding to the entire visual field of the camera. In this way, one is assured that the subject's movement will be recorded at the camera's maximum resolution.

With the development of this automatic movement analysis system, it is possible to do experiments that previously have been avoided because of the cost of filming and the time required for extraction of data.

\section{REFERENCES}

Amassian, V. E., \& Rudell, A. P. When does the cerebellum become important in co-ordinating placing movements? Journal of Physiology, 1978, 276, 35P-36P.

Brugaer, W., \& Milner, M. Computer-aided tracking of body motions using a c.c.d.-image sensor. Medical and Biological Engineering and Computing, 1978, 16, 207-210.

Eaton, R. C., Lavenden, W. A., \& Wieland, C. M. Identification of Mauthner-initiated response patterns in goldfish: Ev- idence from simultaneous cinematography and electrophysiology. Journal of Comparative Physiology, 1981, 144, 521-531.

HALL, J. A. Evaluation of direct-view imaging devices. In L. M. Biberman \& S. Nudelman (Eds.), Photoelectric imaging devices (Vol. 2). New York: Plenum Press, 1971.

MUYBRIDGe, E. Animal locomotion. In Muybridge's complete human and animal locomotion. New York: Dover, 1979. (Animal Locomotion originally published, 1887.)

SchleIDT, W. M. How "fixed" is the action pattern? Zeitschrift für Tierpsychologie, 1974, 36, 184-211.

Rudell, A. P. The television camera used to measure movement. Behavior Research Methods \& Instrumentation, 1979, 11, 339-341.

Wieland, C. M., \& Eaton, R. C. A solid-state camera system for computerized collection and analysis of high-speed movement patterns. Neuroscience Abstracts, 1983, 13, 636.

Winter, D. A., Hobson, D. A., \& Greenlaw, R. K. TVcomputer analysis of kinematics of human gait. Computers and Biomedical Research, 1972, 5, 489-509.

(Manuscript received May 6, 1983; revision accepted for publication August 4, 1983.) 